\title{
Dynamical Structure of Irregular Constrained Systems
}

\author{
Olivera Miškovićc ${ }^{* \dagger}$ and Jorge Zanelli* \\ * Centro de Estudios Científicos (CECS), Casilla 1469, Valdivia, Chile. \\ ${ }^{\dagger}$ Departamento de Física, Universidad de Santiago de Chile, \\ Casilla 307, Santiago 2, Chile.
}

\begin{abstract}
Hamiltonian systems with functionally dependent constraints (irregular systems), for which the standard Dirac procedure is not directly applicable, are discussed. They are classified according to their behavior in the vicinity of the constraint surface into two fundamental types. If the irregular constraints are multilinear (type I), then it is possible to regularize the system so that the Hamiltonian and Lagrangian descriptions are equivalent. When the constraints are power of a linear function (type II), regularization is not always possible and the Hamiltonian and Lagrangian descriptions may be dynamically inequivalent. It is shown that the inequivalence between the two formalisms can occur if the kinetic energy is an indefinite quadratic form in the velocities. It is also shown that a system of type I can evolve in time from a regular configuration into an irregular one, without any catastrophic changes. Irregularities have important consequences in the linearized approximation to nonlinear theories, as well as for the quantization of such systems. The relevance of these problems to Chern-Simons theories in higher dimensions is discussed.
\end{abstract}

\section{Introduction}

Dirac's Hamiltonian analysis provides a systematic method for finding the gauge symmetries and the physical degrees of freedom of constrained systems like gauge theories and gravity [1]. Constraints arise through conditions of the form

$$
\phi^{r}(z) \approx 0 \quad(r=1, \ldots, R)
$$

where $z$ are local coordinates in phase space $\Gamma$. In the most common cases of physical interest the $\phi$ 's are functionally independent; these are the regular constrained systems. There are some exceptional cases in which functional independence is violated. In these irregular systems it is not always clear how to identify symmetries and true degrees of freedom. Moreover, the Hamiltonian and Lagrangian descriptions may not be equivalent in irregular systems.

Irregular systems are not necessarily intractable nor exotic. A common example is a relativistic massless particle $\left(p^{\mu} p_{\mu}=0\right)$, which is irregular at the origin of momentum space $\left(p^{\mu}=0\right)$. There are other physical circumstances in which regularity is violated, and not only for isolated states but on large portions of phase space where the system evolves. This is the case in ChernSimons (CS) theories for dimensions $D \geq 5$ where, for some initial configurations, regularity can fail at all times and one is forced to live with this problem. 
A CS Lagrangian describes a gauge theory for a certain Lie group $G$ in a spacetime of odd dimension. The construction is naturally invariant under diffeomorphisms and provides a nonstandard but otherwise acceptable description of gravity as a gauge theory [2]-[5]. Furthermore, CS theories are highly nonlinear, possess propagating degrees of freedom [6], and have a very rich phase space structure with many different sectors, some of which describe irregular systems [7.

In five-dimensional CS supergravity, it was observed that the linearized action around a certain anti-de Sitter background seems to have one more degree of freedom than the fully nonlinear system [8]. This paradoxical behavior can be seen to arise from a violation of the regularity conditions among the symmetry generators of the theory in the region of phase space defined by the selected background.

Here we address different scenarios in which regularity conditions can be violated, how such systems can be handled in some cases, and why linearization may fail to approximate a nonlinear system faithfully. It is found that there are two main types of irregular constraints, multilinear (type I) and nonlinear (type II). The constraints of the first type can always be regularized, while type II constraints (of the form $f^{k}$, where $f$ has a simple zero and $k>1$ ) can be regularized only if $f$ is a second class function.

Constraints satisfying regularity conditions are sometimes referred to as effective constraints 9]. The issue of regularity (effectiveness) and its relevance for the equivalence between the Lagrangian and Hamiltonian formalisms has also been discussed in [10, 11.

\section{Regularity conditions}

Consider a dynamical system in a phase space $\Gamma$ with local coordinates $z^{i} \equiv(q, p)(i=1, \ldots, 2 n)$. Conditions (1) define the constraint surface

$$
\Sigma=\left\{\bar{z} \in \Gamma \mid \phi^{r}(\bar{z})=0(r=1, \ldots, R)(R \leq 2 n)\right\} .
$$

Dirac's procedure guarantees that the system remains on the constraint surface during its evolution (for reviews, see Refs. [1, [12]-16]). Choosing different coordinates on $\Gamma$ may lead to different forms for the constraint functions whose functional independence is not obvious. The regularity conditions were designed by Dirac to test this [17.

Regularity conditions (RCs): The constraints $\phi^{r} \approx 0$ are regular if and only if their small variations $\delta \phi^{r}$ evaluated on $\Sigma$ define $R$ linearly independent functions of $\delta z^{i}$.

To first order in $\delta z^{i}$, the variations of the constraints have the form

$$
\delta \phi^{r}=J_{i}^{r} \delta z^{i} \quad(r=1, \ldots, R),
$$

where $\left.J_{i}^{r} \equiv \frac{\partial \phi^{r}}{\partial z^{i}}\right|_{\Sigma}$ is the Jacobian evaluated on the constraint surface. An equivalent definition of the RCs is [13]: The set of constraints $\phi^{r} \approx 0$ is regular if and only if the Jacobian $J_{i}^{r}=\left.\frac{\partial \phi^{r}}{\partial z^{i}}\right|_{\Sigma}$ has maximal rank, $\Re(\mathbf{J})=R$.

A simple classical mechanical example of functionally dependent constraints occurs in a 2dimensional phase space with coordinates $(q, p)$ and constraints $\phi^{1} \equiv q \approx 0$ and $\phi^{2} \equiv p q \approx 0$. In this case, $\mathbf{J}=\left[\begin{array}{ll}1 & p \\ 0 & q\end{array}\right]_{q=0}$ and $\Re(\mathbf{J})=1$. 
A system of just one constraint can also fail the test of regularity. Consider for example the constraint $\phi=q^{2} \approx 0$ in a 2-dimensional phase space. In this case, $\mathbf{J}=(2 q, 0)_{q^{2}=0}=0$ and $\Re(\mathbf{J})=0$. The same problem occurs with the constraint $q^{k} \approx 0$, for $k>1$, which has a zero of $k$-th order on the constraint surface. This example illustrates that one constraint may be dependent on itself, while one function is, by definition, always functionally independent.

Equivalence: Different sets of constraints are said to be equivalent if they define the same constraint surface.

Note that this definition refers to the locus of constraints, not to equivalence of the resulting dynamics. Since the surface $\Sigma$ is defined by the zeros of the constraints, while the regularity conditions depend on their derivatives, it is possible to replace a set of irregular $\phi$ 's by an equivalent set of regular constraints $\tilde{\phi}$.

In the classification of irregular systems, two questions present themselves: what is the nature of the constraints that give rise to irregularity, and where the irregularities can occur. These issues are addressed in the following subsections. A third question is whether a system can evolve from an initial state in which regularity holds, into an irregular configuration. This will be discussed in the last section.

\subsection{Basic types of irregular constraints}

Irregular constraints can be classified according to their behavior in the vicinity of the surface $\Sigma$. For example, linearly dependent constraints have Jacobian with constant rank $R^{\prime}$ throughout $\Sigma$, and

$$
\phi^{r} \equiv J_{i}^{r}(\bar{z})\left(z^{i}-\bar{z}^{i}\right) \approx 0, \quad \Re(\mathbf{J})=R^{\prime}<R .
$$

These constraints are regular systems in disguise simply because $R-R^{\prime}$ constraints are redundant and should be discarded. The subset with $R^{\prime}$ linearly independent constraints gives the correct description. For example, the linearly dependent constraints $\phi^{1}=z$ and $\phi^{2}=2 z$ are clearly in this category. Apart from this trivial case, two main types of truly irregular constraints, which do not possess a linear approximation in the vicinity of some points of $\Sigma$, can be distinguished:

Type I. Multilinear constraints. Consider the constraint

$$
\phi \equiv \prod_{i=1}^{M} f_{i}(z) \approx 0,
$$

where the functions $f_{i}$ have simple zeros. Each factor defines a surface of codimension 1 ,

$$
\Sigma_{i} \equiv\left\{\bar{z} \in \Gamma \mid f_{i}(\bar{z})=0\right\}
$$

and $\Sigma$ is the collection of all surfaces, $\Sigma=\bigcup \Sigma_{i}$. The rank of Jacobian of $\phi$ is reduced at intersections

$$
\Sigma_{i j} \equiv \Sigma_{i} \bigcap \Sigma_{j}
$$

Thus, the RCs hold everywhere on $\Sigma$, except at the intersections $\Sigma_{i j}$, where $\phi$ has zeros of higher order. Note that the intersections (7) also include the points where more than two $\Sigma$ 's overlap. 
Type II. Nonlinear constraints. Consider the constraint of the form

$$
\phi \equiv[f(z)]^{k} \approx 0 \quad(k>1),
$$

where the function $f(z)$ has a simple zero. This constraint has a zero of order $k$ in the vicinity of $\Sigma$, its Jacobian vanishes on the constraint surface and, therefore, the RCs fail (here we assume $k>1$ in order to avoid infinite values for $\frac{\partial \phi}{\partial z^{i}}$ on $\Sigma$ ). It could seem harmless to replace $\phi$ by the equivalent regular constraint $f(z) \approx 0$, but it turns out that this may change the dynamics of original system, as we show below.

Types I and II are the two fundamental generic classes of irregular constraints. In general, there can be combinations of them occurring simultaneously near a constraint surface, as in constraints of the form $\phi=\left[f_{1}(z)\right]^{2} f_{2}(z) \approx 0$, etc.

\subsection{Classification of constraint surfaces}

The previous classification refers to the way in which $\phi$ approaches zero. Now we will discuss where regularity can be violated. The rank of the Jacobian $\frac{\partial \phi^{r}}{\partial z^{i}}$ need not be constant throughout $\Sigma$ : suppose one eigenvalue of the Jacobian vanishes on a submanifold $\Sigma_{0} \subset \Sigma$. On $\Sigma_{0}$ regularity is violated, while it still holds on the rest of $\Sigma$. Thus, barring accidental degeneracies such as linearly dependent constraints, one of these three situation may present themselves:

A. The RCs are satisfied everywhere on the constraint surface: $\mathbf{J}$ has maximal rank throughout $\Sigma$ (regular systems).

B. The RCs fail everywhere on the constraint surface: $\mathbf{J}$ has constant $\operatorname{rank} R^{\prime}<R$ on $\Sigma$.

C. The RCs fail on $\Sigma_{0}:\left.\Re(\mathbf{J})\right|_{\Sigma_{0}}=R^{\prime}<R$, while $\Re(\mathbf{J})=R$ elsewhere on $\Sigma$.

In the last case, the constraint surface can be decomposed into two non overlapping sets $\Sigma_{0}$ and $\Sigma_{R}$. Then, the rank of the Jacobian jumps from $\Re(\mathbf{J})=R$ on $\Sigma_{R}$, to $\Re(\mathbf{J})=R^{\prime}$ on $\Sigma_{0}$. Although the functions $\phi^{r}$ are continuous and differentiable, this is not sufficient for regularity. Irregular cases are illustrated by the following examples.

In a $(2+N)$-dimensional phase space $\left(q, p, z^{1}, \ldots, z^{N}\right)$, the constraints $\phi^{1} \equiv q-p \approx 0$ and $\phi^{2} \equiv q p \approx 0$ are irregular on the whole constraint surface $\left\{\left(0,0, z^{1}, \ldots, z^{N}\right)\right\}$, where the Jacobian has rank $\Re(\mathbf{J})=1$. Note that these constraints are always irregular, although the functions $q+p$ and $q p$ are functionally independent everywhere except for $q=p$, which happens to be the case at the constraint surface.

An example having both regular and irregular sectors is a massless relativistic particle in Minkowski space with phase space $\left(q^{\mu}, p_{\nu}\right)$. The constraint $\phi \equiv p^{\mu} p_{\mu} \approx 0$ has Jacobian $\mathbf{J}=$ $\left(0,2 p^{\mu}\right)_{\phi=0}$, and its rank is one everywhere, except at the apex of the cone, $p^{\mu}=0$, where the light-cone is not differentiable and the Jacobian has rank zero.

The lack of regularity, however, is not necessarily due to the absence of a well defined smooth tangent space for $\Sigma$. Consider for example the multilinear constraint

$$
\phi(x, y, z)=(x-1)\left(x^{2}+y^{2}-1\right) \approx 0 .
$$

Here the constraint surface $\Sigma$ is composed of two sub-manifolds: the plane $\Pi=\{(x, y, z)$ | $x-1 \approx 0\}$, and the cylinder $C=\left\{(x, y, z) \mid x^{2}+y^{2}-1 \approx 0\right\}$, which are tangent to each other along the line $L=\{(x, y, z) \mid x=1, y=0, z \in \mathbb{R}$. The Jacobian on $\Sigma$ is

$$
\mathbf{J}=\left(3 x^{2}+y^{2}-2 x-1,2 y(x-1), 0\right)_{\phi=0}
$$


and its rank is 1 everywhere, except on $L$, where it is zero. The constraint $\phi$ is irregular on this line. However, the tangent vectors to $\Sigma$ are well defined there. The irregularity arises because $\phi$ is a multilinear constraint of the type described by (5) and has two simple zeros overlapping on $L$. The equivalent set of regular constraints on $L$ is $\left\{\phi_{\Pi}=x-1 \approx 0, \phi_{C}=x^{2}+y^{2}-1 \approx 0\right\}$, as we will see below.

\section{Treatment of irregular systems}

In what follows regular systems and linearly dependent constraints will not be discussed. They are either treated in standard texts, or they can be trivially reduced to the regular case.

\subsection{Multilinear constraints}

Consider a system of type I, as in Eq. (5). In the vicinity of an irregular point where only two surfaces (6) intersect, say $\Sigma_{1}$ and $\Sigma_{2}$, the constraint $\phi \approx 0$ is equivalently described by the set of regular constraints

$$
f_{1} \approx 0, \quad f_{2} \approx 0 .
$$

This replacement generically changes the Lagrangian of the system, and the orbits, as well. Suppose the original canonical Lagrangian is

$$
L(q, u)=p_{i} \dot{q}^{i}-H(q, p)-u \phi(q, p),
$$

where $H$ is the Hamiltonian containing all regular constraints. Replacing $\phi$ by (11), gives rise to an effective Lagrangian

$$
L_{12}(q, v)=p_{i} \dot{q}^{i}-H(q, p)-v^{1} f_{1}(q, p)-v^{2} f_{2}(q, p) .
$$

defined on $\Sigma_{12}$. Thus, instead of the irregular Lagrangian (12) defined on the whole $\Sigma$, there is a collection of regularized effective Lagrangians defined in the neighborhood of the different intersections of $\Sigma_{i}$ s. For each of these regularized Lagrangians, the Dirac procedure can be carried out to the end.

Let us illustrate this with the example of a Lagrangian in a $(2+N)$-dimensional configuration space $\left(x, y, q^{1}, \ldots, q^{N}\right)$,

$$
L=\frac{1}{2} \sum_{k=1}^{N}\left(\dot{q}^{k}\right)^{2}+\frac{1}{2}\left(\dot{x}^{2}+\dot{y}^{2}\right)-\lambda x y .
$$

This Lagrangian describes a free particle moving on the set

$$
\left\{\left(x, y, q^{k}\right) \in \mathbb{R}^{N+2} \mid x y=0\right\} \subset \mathbb{R}^{N+2},
$$

which is the union of two $(N+1)$-dimensional planes where $x$ and $y$ vanish respectively. The constraint surface defined by $x y=0$ can be divided into the following sets:

$$
\begin{aligned}
\Sigma_{1} & =\left\{\left(x, 0, q^{k} ; p_{x}, p_{y}, p_{k}\right) \mid x \neq 0\right\} \\
\Sigma_{2} & =\left\{\left(0, y, q^{k} ; p_{x}, p_{y}, p_{k}\right) \mid y \neq 0\right\} \\
\Sigma_{12} & =\left\{\left(0,0, q^{k} ; p_{x}, p_{y}, p_{k}\right)\right\} .
\end{aligned}
$$


The constraint is regular on $\Sigma_{1} \bigcup \Sigma_{2}$, while on $\Sigma_{12}$ it is irregular and can be exchanged by $\left\{\phi_{1}=x \approx 0, \phi_{2}=y \approx 0\right\}$. The corresponding regularized Lagrangians are

$$
\begin{aligned}
L_{1} & =\frac{1}{2} \sum_{k=1}^{N}\left(\dot{q}^{k}\right)^{2}+\frac{1}{2} \dot{x}^{2}, \\
L_{2} & =\frac{1}{2} \sum_{k=1}^{N}\left(\dot{q}^{k}\right)^{2}+\frac{1}{2} \dot{y}^{2} \\
L_{12} & =\frac{1}{2} \sum_{k=1}^{N}\left(\dot{q}^{k}\right)^{2}
\end{aligned}
$$

and the Lagrange multipliers have dropped out, so the regularized Lagrangians describe physical degrees of freedom only - as expected.

The corresponding regularized Hamiltonians are

$$
\begin{aligned}
H_{1} & =\frac{1}{2} \sum_{k=1}^{N} p_{k}^{2}+\frac{1}{2} p_{x}^{2} \\
H_{2} & =\frac{1}{2} \sum_{k=1}^{N} p_{k}^{2}+\frac{1}{2} p_{y}^{2} \\
H_{12} & =\frac{1}{2} \sum_{k=1}^{N} p_{k}^{2},
\end{aligned}
$$

which are defined in the corresponding reduced manifolds of phase space (obtained after completing the Dirac procedure):

$$
\begin{aligned}
\tilde{\Sigma}_{1} & =\left\{\left(x, 0, q^{k} ; p_{x}, 0, p_{k}\right) \mid x \neq 0\right\} \\
\tilde{\Sigma}_{2} & =\left\{\left(0, y, q^{k} ; 0, p_{y}, p_{k}\right) \mid y \neq 0\right\} \\
\tilde{\Sigma}_{12} & =\left\{\left(0,0, q^{k} ; 0,0, p_{k}\right)\right\} .
\end{aligned}
$$

It is straightforward to generalize the proposed treatment when more than two surfaces $\Sigma_{i}$ overlap.

Evolution of a multilinearly constrained system. Since in the presence of a multilinear constraint there are regions of the phase space where the Jacobian has different rank, a question arises about the evolution of this system. Can the system evolve from a generic configuration in a region of maximal rank, reaching a configuration of lower rank in finite time? In the case that that were possible, what happens with the system afterwards?. (This problem should not be confused with the issues arising in degenerate systems [18]- 20].)

To answer this question let us consider the simple example discussed above (14), for $N=1$,

$$
L=\frac{1}{2}\left(\dot{x}^{2}+\dot{y}^{2}+\dot{z}^{2}\right)-\lambda x y .
$$


In the regions $\Sigma_{1}$ and $\Sigma_{2}$ [see Eqs. (16)], the rank is maximal and the free particle can move freely along the $x$ - or $y$-axis respectively.

Suppose that the initial state is

$$
x(0)=a>0, \quad y(0)=0, \quad z(0)=0, \quad \dot{x}(0)=-v<0, \quad \dot{y}(0)=0, \quad \dot{z}(0)=0,
$$

so that the particle is moving on $\Sigma_{1}$, with finite speed along the $x$-axis towards $x=0$ on $\Sigma_{12}$. The evolution is given by $\bar{x}(t)=a-v t, \bar{y}(t)=0, \bar{z}(t)=0$ and the particle clearly reaches $x=0$ in a finite time $(T=a / v)$. What happens then? According to the evolution equation, for $x<0$ the trajectory takes the form $\bar{x}(t)=a^{\prime}-v^{\prime} t, \bar{y}(t)=0, \bar{z}(t)=0$, however the action would be infinite unless $a=a^{\prime}$ and $v=v^{\prime}$. Therefore, the particle continues unperturbed past beyond the point where the RCs fail. So, the irregular surface is not only reachable in a finite time, but it is crossed without any observable effect on the trajectory.

¿From the point of view of the trajectory in phase space, it is clear that the initial state $(a, 0,0 ;-v, 0,0)$ lies on the surface $\tilde{\Sigma}_{1}$, and at $t=T$ the system reaches the point $(0,0,0 ;-v, 0,0)$, which does not lie on the surface $\tilde{\Sigma}_{12}=\left\{\left(0,0, z ; 0,0, p_{z}\right)\right\}$.

While it is true that at $t=T$ the Jacobian changes rank, it would be incorrect to conclude that the evolution suffers a jump since the dynamical equations are perfectly valid there. In order to have significant change in dynamics, the Jacobian should change its rank in an open set.

\section{$3.2 \quad$ Nonlinear constraints}

Let us now turn to the case of irregular systems of type II. As we will show, it is possible to replace a nonlinear irregular constraint by an equivalent linear one without changing the dynamical contents of the theory, provided the linear constraint is second class. Otherwise, the resulting Hamiltonian dynamics will be, in general, inequivalent to that of the original Lagrangian system.

In order to illustrate this point, consider a system given by the Lagrangian

$$
L(q, u)=\frac{1}{2} \gamma_{i j} \dot{q}^{i} \dot{q}^{j}-u[f(q)]^{k},
$$

where $k>1$ and

$$
f(q) \equiv c_{i} q^{i} \neq 0, \quad i=1, \ldots, N .
$$

Here we assume the metric $\gamma_{i j}$ to be constant and invertible, and the coefficients $c_{i}$ are also constant. The Euler-Lagrange equations describe a free particle in an $N$-dimensional space, with time evolution $\bar{q}^{i}(t)=v_{0}^{i} t+q_{0}^{i}$, where $u(t)$ is a Lagrange multiplier. This solution is determined by $2 N$ initial conditions, $q^{i}(0)=q_{0}^{i}$ and $\dot{q}^{i}(0)=v_{0}^{i}$ subject to the constraints $c_{i} q_{0}^{i}=0$ and $c_{i} v_{0}^{i}=0$. Thus, the system possesses $N-1$ physical degrees of freedom.

In the Hamiltonian approach this system has a primary constraint $\pi \equiv \frac{\partial L_{2}}{\partial \dot{u}} \approx 0$ whose preservation in time leads to the secondary constraint

$$
\phi \equiv[f(q)]^{k} \approx 0 .
$$


According to (8), this is a nonlinear constraint and there are no further constraints. As a consequence, the system has only two first class constraints $\left\{\pi \approx 0, f^{k} \approx 0\right\}$, and $N-1$ degrees of freedom, as found in the Lagrangian approach.

On the other hand, if one chooses instead of (24), the equivalent linear constraint

$$
f(q)=c_{i} q^{i} \approx 0
$$

then its time evolution yields a new constraint,

$$
\chi(p) \equiv \gamma^{i j} c_{i} p_{j} \approx 0
$$

Now, since

$$
\{f, \chi\}=\gamma^{i j} c_{i} c_{j} \equiv\|c\|^{2}
$$

two cases can be distinguished:

- If $\|c\|=0$, there are three first class constraints, $\pi \approx 0, f \approx 0$ and $\chi \approx 0$, which means that the system has $N-2$ physical degrees of freedom. In this case, substitution of (24) by the equivalent linear constraint (25), yields a dynamically inequivalent system.

- If $\|c\| \neq 0$, then $f \approx 0$ and $\chi \approx 0$ are second class, while $\pi \approx 0$ is first class, which leaves $N-1$ physical degrees of freedom and the substitution does not change the dynamics of the system.

Thus, if $f^{k} \approx 0$ is irregular, replacing it by the regular constraint $f \approx 0$ changes the dynamics if $f$ is a first class function, but it gives the correct result if it is a second class function.

Note that in the Lagrangian description there is no room to distinguish first and second class constraints, so it would seem like the value of $\|c\|$ didn't matter. However, the inequivalence of the substitution can be understood in the Lagrangian analysis as well. Suppose that it were permissible to exchange the constraint $f^{k} \approx 0$ by $f \approx 0$ in the Lagrangian. Then, instead of (22), one would have

$$
\tilde{L}(q, u)=\frac{1}{2} \gamma_{i j} \dot{q}^{i} \dot{q}^{j}-u f(q) .
$$

It can be easily checked that (28) yields $N-2$ degrees of freedom when $\|c\|=0$, and $N-1$ degrees of freedom when $\|c\| \neq 0$, which agrees with the results obtained in the Hamiltonian analysis. Note that the substitution of $f^{k}$ by $f$ modifies the dynamics only if $\gamma^{i j} c_{i} c_{j}=0$, but this can happen nontrivially only if the metric $\gamma_{i j}$ is not positive definite.

In general, a nonlinear irregular constraint $\phi \approx 0$ has a multiple zero on the constraint surface $\Sigma$, which means that its gradient vanishes on $\Sigma$ as well. An immediate consequence of $\left(\partial \phi / \partial z^{i}\right) \approx 0$, is that $\phi$ commutes with all finite functions on $\Gamma$,

$$
\{\phi, F(z)\} \approx 0 .
$$

As a consequence, $\phi \approx 0$ is first class and is always preserved in time,

$$
\dot{\phi} \approx 0 \text {. }
$$


On the other hand, a nonlinear constraint cannot be viewed as a symmetry generator simply because it does not generate any transformation,

$$
\delta_{\varepsilon} z^{i}=\left\{z^{i}, \varepsilon \phi\right\} \approx 0
$$

Consistently with this, $\phi$ cannot be gauge-fixed, as there is no finite function $\mathcal{P}$ on $\Gamma$ such that

$$
\{\phi, \mathcal{P}\} \neq 0 \text {. }
$$

In this sense, a nonlinear first class constraint that cannot be gauge-fixed, cancels only half a degree of freedom. The other half degree of freedom cannot be cancelled because the gauge-fixing function does not exist and, in particular, it cannot appear in the Hamiltonian. Although the features (29] 322) allow counting the degrees of freedom in a theory, these systems are pathological and their physical relevance is questionable since their Lagrangians cannot be regularized.

When a nonlinear constraint $\phi \approx 0$ can be exchanged by a regular one, the Lagrangian is regularized as in the case of multilinear constraints. For example, the system (22) with $\|c\| \neq 0$ has Hamiltonian

$$
H=\frac{1}{2} \gamma^{i j} p_{i} p_{j}+\lambda \pi+u f(q)
$$

where $f=c_{i} q^{i}$ will turn out to be a second class constraint. The corresponding regularized Lagrangian is

$$
L_{r e g}=\frac{1}{2} \gamma_{i j} \dot{q}^{i} \dot{q}^{j}-u f(q)
$$

which coincides with $\tilde{L}$, Eq. (28), as expected.

In the Refs. 10, 11] irregular systems of the type II were discussed. It was pointed out that there was a possible loss of dynamical information in some cases. From our point of view, it is clear that this would occur when $f$ is a first class function.

\section{Linearization of irregular systems}

It has been observed in five dimensional Chern Simons theory, that the effective action for the linearized perturbations of the system around certain backgrounds seems to have more degrees of freedom than the fully nonlinear theory [8]. This is puzzling since the heuristic picture is that the degrees of freedom of a system correspond to the small perturbations around a local minimum of the action, and therefore the number of degrees of freedom should not change when the linearized approximation is used.

In view of the discussion in the previous section, it is clear that a possible solution of the puzzle lies in the fact that substituting a nonlinear constraint by a linear ones may change the dynamical features of the theory. But the problem with linear approximations is more serious: the linearized approximation retains only up to quadratic and bilinear terms in the Lagrangian, which give linear equations for the perturbations. Thus, irregular constraints in the vicinity of the constraint surface are erased in the linearized action. The smaller number of constraints in the effective theory can lead to the wrong conclusion that the effective system possess more degrees of freedom than the unperturbed theory. The lesson to be learned is that the linear approximation is not valid in the part of the phase space where the RCs fail. 
This is illustrated by the same example discussed earlier (22). One can choose as a background the solution $\left(\bar{q}^{1}, \ldots, \bar{q}^{N}, \bar{u}\right)$, where $\bar{q}^{i}(t)=q_{0}^{i}+v_{0}^{i} t$ satisfies the constraint

$$
c_{i} \bar{q}^{i}=0
$$

and $\bar{u}(t)$ is an arbitrarily given function. This describes a free particle moving in the $(N-1)$ dimensional plane defined by (35). The linearized effective Lagrangian, to second order in the small perturbations $s^{i}=q^{i}-\bar{q}^{i}(t)$ and $w=u-\bar{u}(t)$, has the form

$$
L_{e f f}(s, w)=\frac{1}{2} \gamma_{i j}\left(v_{0}^{i}+\dot{s}^{i}\right)\left(v_{0}^{j}+\dot{s}^{j}\right)-\bar{u}\left(c_{i} s^{i}\right)^{2},
$$

and the equations of motion are

$$
\ddot{s}^{i}+\Gamma_{j}^{i}(t) s^{j}=0 \quad i=1, \ldots, N,
$$

where $\Gamma_{j}^{i} \equiv 2 \bar{u} \gamma^{i k} c_{k} c_{j}$ is the eigen frequency matrix. Since $\bar{u}$ is not a dynamical variable, it is not varied and the nonlinear constraint $\left(c_{i} s^{i}\right)^{2}=0$ is absent from the linearized equations. The system described by (37) possesses $N$ physical degrees of freedom, that is, one degree of freedom more than the original nonlinear theory (22).

The only indication that one of these degrees of freedom has a nonphysical origin is the following: If $\|c\| \neq 0$, splitting the components of $s^{i}$ along $c_{i}$ and orthogonal to $c_{i}$ as

$$
s^{i}(t) \equiv s(t) \gamma^{i j} c_{j}+s_{\perp}^{i}(t)
$$

gives rise to the projected equations

$$
\begin{aligned}
\ddot{s}_{\perp}^{i} & =0 \\
\ddot{s}+2 \bar{u}(t)\|c\|^{2} s & =0 .
\end{aligned}
$$

The $N-1$ components of $s_{\perp}^{i}(t)$ obey a deterministic second order equation, whereas $s(t)$ satisfies an equation which depends on an indeterminate arbitrary function $\bar{u}(t)$. The dependence of $s=\bar{s}(t, \bar{u}(t))$ on the background Lagrange multiplier $\bar{u}$ is an indication that $s$ is a nonphysical degree of freedom, since $u$ was an arbitrary function to begin with. This is not manifest in Eq. (40), where $\bar{u}$ is a fixed function and, from a naive point of view, $s(t)$ is determined by the same equation, regardless of the physically obscure origin of the function $\bar{u}$. It is this naive analysis that leads to the wrong conclusion indicated above.

Let us emphasize that a linearized theory may be consistent by itself, but it is not necessarily a faithful approximation of a nonlinear theory.

One way to avoid the inconsistencies between the original theory and the linearized one would be to first regularize the constraints (if possible) and then linearize the corresponding regular Lagrangian.

\section{Chern-Simons theories}

Hamiltonian structure of CS theories has been studied in 6]. The phase space of a CS theory in $D=2 n+1$ space-time dimensions, invariant under $N$-parameter gauge group, is defined 
by canonically conjugate pairs of fields $\left(A_{i}^{a}(x, t), \pi_{a}^{i}\left(x^{\prime}, t\right)\right)$, where $a=1, \ldots, N$ and $x^{i}(i=$ $1, \ldots, 2 n)$ are the local coordinates on a spatial section. The CS Hamiltonian density is given by

$$
\mathcal{H}=A_{0}^{a} \tilde{G}_{a}+u_{i}^{a} \phi_{a}^{i},
$$

where $u_{i}^{a}(x, t)$ and $A_{0}^{a}(x, t)$ are Lagrange multipliers for the constraints

$$
\begin{aligned}
\phi_{a}^{i} & \equiv \pi_{a}^{i}-\mathcal{L}_{a}^{i}\left(A_{j}^{b}\right) \approx 0 \\
G_{a} & =g_{a a_{1} \cdots a_{n}} F^{a_{1}} \wedge \cdots \wedge F^{a_{n}} \approx 0
\end{aligned}
$$

Here $G_{a} \equiv d^{2 n} x \tilde{G}_{a}, g$ is a symmetric tensor of rank $n+1$, invariant under action of a gauge group, and $F=d A+A \wedge A$ is the curvature 2-form associated to the gauge field 1-form $A$.

Constraints $\phi_{a}^{i}$ are regular because they are linear in momenta. Thus, the regularity of CS theories is determined by momentum-independent constraints $G_{a}$. Their small variations, $\delta G_{a}=\mathbf{J}_{a b} D \delta A^{b}$, evaluated at $G_{a}=0$, give the $(2 n-2)$-form $\mathbf{J}_{a b}$, which can be identified as the Jacobian,

$$
\mathbf{J}_{a b} \equiv n g_{a b a_{2} \cdots a_{n}} F^{a_{2}} \wedge \cdots \wedge F^{a_{n}} .
$$

According to Dirac's definition, sufficient and necessary condition for $G_{a}$ to be regular is

$$
\Re\left(\mathbf{J}_{a b}\right)=N .
$$

Since $\mathbf{J}_{a b}$ is field dependent, its rank may change in space. In particular, for a pure gauge configuration $F=0$, and $\mathbf{J}_{a b}$ has rank zero. For other configurations, the rank of Jacobian can range from zero to $N$, and the irregularities are always of multilinear type because in the expression (43) the phase space coordinates $A_{i}^{a}$ occur only linearly.

In general, a non-abelian CS theory for $D \geq 5$ possesses a non-vanishing number of physical degrees of freedom [6]

$$
f_{2 n+1}(N)=n N-n-N \quad(N \geq 2)
$$

in regular and generic cases 20]. Therefore, in CS theories, the study of dynamics requires not only the analysis of regularity, but also of genericity. In spite of the fact that both conditions are expressed in terms of the same matrix $\Omega_{a b}^{i j}$, they are independent. For example, the extreme case of $F=0$ is both irregular and degenerate, but there are examples in CS supergravity which are generic and irregular [8]. The opposite case occurs in a five-dimensional CS theory based on $G_{1} \times G_{2}$ for particular choice of invariant tensor. In this case, there exist configurations which are regular but degenerate. Take the group indices as $a=(r, \alpha)$ corresponding to $G_{1}$ and $G_{2}$ respectively, and invariant tensor as $g_{r s 1}=g_{r s}$ and $g_{\alpha \beta \overline{1}}=g_{\alpha \beta}$ (both invertible). Then the configuration $F^{a}=\left(f^{1} d x^{1} \wedge d x^{2}, h^{\overline{1}} d x^{3} \wedge d x^{4}\right)$ is regular and degenerate. Indeed, $\mathbf{J}_{a b}=$ $\left(\begin{array}{cc}g_{r s} f^{1} & 0 \\ 0 & g_{\alpha \beta} h^{\overline{1}}\end{array}\right)$ is regular, while $\Omega_{a b}^{i j}$ with non-vanishing components $\Omega_{r s}^{34}=g_{r s} f^{1}$ and $\Omega_{\alpha \beta}^{12}=$ $g_{\alpha \beta} h^{\overline{1}}$ has $2 N$ zero modes and is therefore degenerate.

As a consequence of existence of both regularity and genericity issues, the regularization problem is much more delicate in CS theories. 


\section{Comments}

1 - Dirac conjecture. Dirac conjectured that all first class constraints generate gauge symmetries [17. It was shown that Dirac's conjecture is not true for first class constraints of the form $f^{k}(k>1)$, and following from $\dot{f} \approx 0[14,15]$. Therefore, for systems with nonlinear constraints, the conjecture does not work and they generically provide counterexamples of it [13, 21, 22.

From the point of view of irregular systems, it is clear that Dirac's conjecture fails for nonlinear constraints because they do not generate any local transformation, c.f. Eq. (31). In Refs. 10, 11] it was observed that Dirac's conjecture may not hold in the presence of irregular constraints of type II.

In the case of multilinear constraints, however, Dirac's conjecture holds. The fact that at irregular points the constraints do not generate any transformation only means that these are fixed points of the gauge transformation.

2 - Quantization. Although, in view of the above discussion, it is possible to deal systematically with classical theories containing irregular constraints, there may be severe problems in their quantum description. Consider a path integral of the form

$$
Z \sim \int[d q][d p][d u] \exp i[p \dot{q}-H(q, p)-u \phi(q, p)]
$$

where $\phi=[f(q, p)]^{k}$ is a nonlinear constraint. Integration on $u$ yields to $\delta\left(f^{k}\right)$, which is not well-defined for a zero of order $k>1$, making the quantum theory ill defined. Only if the nonlinear constraint could be exchanged by the regular one, $f(q, p) \approx 0$, the quantum theory could be saved. An example of this occurs in the standard approach for QED, where it is usual practice to introduce the nonlinear gauge fixing term $u\left(\partial_{i} A^{i}\right)^{2}$ in order to fix the primary constraint $\pi^{0} \equiv\left(\delta I_{E D} / \delta \dot{A}_{0}\right) \approx 0$. Since the gauge condition $f(A)=\partial_{i} A^{i}(x) \approx 0$ is a second class constraint, its substitution by a regular constraint does not change its dynamical structure.

\section{Summary}

We have discussed the dynamics and evolution of a system possessing constraints which may violate the regularity conditions (functional independence) on some subsets of the constraint surface $\Sigma$. These so-called irregular systems are seen to arise generically because of nonlinearities in the constraints and can be classified into two families: multilinear (type I) and nonlinear (type II).

- Type I constraints are of the form $\phi=\prod f_{i}(z)$, where $f_{i}$ possess simple zeros. These constraints violate the regularity conditions (RCs) on sets of measure zero on the constraint surface $\Sigma$.

- Type I constraints can be exchanged by equivalent constraints which are regular giving an equivalent dynamical system. 
- Type II constraints are of the form $\phi=f^{k}(k>1)$ where $f$ has a simple zero. They violate the RCs on sets of nonzero measure on $\Sigma$.

- A type II constraint can be replaced by an equivalent linear one only if the latter is second class; if the equivalent linear constraint is first class, substituting it for the original constraint would change the system.

- In general, the orbits can cross the configurations where the RCs are violated without any catastrophic effect for the system. If the symplectic form degenerates at the irregular points, additional analysis is required.

- The naive linearized approximation of an irregular constrained system generically changes it by erasing the irregular constraints. In order to study the perturbations around a classical orbit in an irregular system, it would be necessary to first regularize it (if possible) and only then do the linearized approximation.

- Chern-Simons theories possess irregular and regular sectors. This problem is independent of the presence of degeneracies in the symplectic form, making the regularization problem much more complex.

\section{Acknowledgments}

We are grateful to Milutin Blagojević, Marc Henneaux and Claudio Teitelboim for enlightening and helpful comments, and specially to Ricardo Troncoso for many useful discussions, insights and friendly criticisms. We would also like to thank Josep María Pons and Antonio GarcíaZenteno for bringing their work to our attention and for indicating its relevance to ours. This work is partially funded by grants FONDECYT 1020629, 7020629, 1010450, 2010017 and the grant MECESUP USA 9930. One of us (O. M.) thanks the Abdus Salam ICTP for hospitality during the completion of this work. The generous support of Empresas CMPC to CECS is also acknowledged. CECS is a Millennium Science Institute and is funded in part by grants from Fundación Andes and the Tinker Foundation.

\section{References}

[1] P. A. M. Dirac, Lectures on Quantum Mechanics (Yeshiva University, New York, 1964).

[2] A. Chamseddine, Phys. Lett. B 233 (1989) 291; Nucl. Phys. B 346 (1990) 213.

[3] R. Floreanini and R. Percacci, Phys. Lett. B 224 (1989) 291.

[4] V. P. Nair and J. Schiff, Phys. Lett. B 246 (1990) 423; Nucl. Phys. B 371 (1992) 329.

[5] R. Troncoso and J. Zanelli, Phys. Rev. D 58: R101703 (1998); Int. J. Theor. Phys. 38 (1999) 1181.

[6] M. Bañados, L. J. Garay and M. Henneaux, Phys. Rev. D 53 (1996) 593; Nucl. Phys. B 476 (1996) 611. 
[7] O. Mišković and J. Zanelli, Irregular Hamiltonian Systems, Proceedings of the XIII Chilean Symposium of Physics, Concepción, Chile, November 2002. E-print Archive: hep-th/0301256

[8] O. Chandía, R. Troncoso and J. Zanelli, Dynamical Content of Chern-Simons Supergravity, Talk given at The Second La Plata Meeting on Trends in Theoretical Physics, Buenos Aires, Argentina, 28 Nov - 4 Dec 1998. E-print Archive: hep-th/9903204.

[9] C. Batlle, J. Gomis, J. M. Pons and N. Roman-Roy, J. Math. Phys. 27 (1986) 2953.

[10] J. A. García and J. M. Pons, Int. J. Mod. Phys. A13 (1998) 3691.

[11] J. M. Pons, D. C. Salisbury and L. C. Shepley, Phys. Rev. D62 (2000) 064024.

[12] A. J. Hanson, T. Regge and C. Teitelboim, Constrained Hamiltonian Systems (Accademia Nazionale dei Lincei, Rome, 1976).

[13] M. Henneaux and C. Teitelboim, Quantization of Gauge Systems (Princeton University Press, Princeton, 1992).

[14] L. Castellani, Ann. Phys. (N. Y.) 143 (1982) 357.

[15] M. Blagojević, Gravity and Gauge Symmetries (Institute of Physics Publishing, London, 2001).

[16] N. P. Chitaia, S. A. Gogilidze and Yu. S. Surovtsev, Phys. Rev. D 56 (1997) 1135; Phys. Rev. D 56 (1997) 1142.

[17] P. A. M. Dirac, Can. J. Math. 3 (1951) 1.

[18] These questions have a similar ring to those presented in the case of a degenerate dynamical system, in which the symplectic matrix changes its rank [19]: Is it possible for a system in a generic initial configuration, to reach (in a finite time) a configuration where the symplectic form has lower rank? That problem is unrelated to the one discussed here and can be treated independently. In the present case it is the functional independence of the constraints that fails; in the degenerate dynamical systems it is the symplectic structure that breaks down.

[19] J. Saavedra, R. Troncoso and J. Zanelli, J. Math. Phys. 42 (2001) 4383.

[20] A configuration is called generic if the symplectic form $\Omega$ is invertible. Otherwise, it is called degenerate. In the case of CS theories, the symplectic form is given by $d^{2 n} x \Omega_{a b}^{i j} \equiv$ $d x^{i} \wedge d x^{j} \wedge J_{a b}$. In these theories, the generic configurations are those for which $(i)$ the $2 n$ independent vectors $\left(\mathbf{V}_{i}\right)_{j}^{a}=F_{i j}^{a}$ are the only zero modes of $\Omega_{a b}^{i j}$, and $(i i)$ the rank of $\Omega_{a b}^{i j}$ is largest, $\Re\left(\Omega_{a b}^{i j}\right)=2 n N-2 n$.

[21] T. R. Allcock, Phil. Trans. R. Soc. (Lond.) 279 (1975) 33.

[22] R. Cawley, Phys. Rev. Lett. 42 (1979) 413. 